\title{
Percepções de enfermeiros sobre gestão do cuidado e seus fatores intervenientes para o controle da tuberculose
}

\author{
Nurses' perceptions on care management and its intervening factors for tuberculosis control \\ Percepciones de enfermeros sobre gestión de la atención y factores intervinientes para el control de la \\ tuberculosis
}

Fabiane Oliveira da Silva ${ }^{1}$ (B) Ivaneide Leal Ataíde Rodrigues ${ }^{2}$ (1)

Alexandre Aguiar Pereira ${ }^{1}$ (D)

Laura Maria Vidal Nogueira ${ }^{2}$ (1)

Erlon Gabriel Rego de Andrade ${ }^{1}$ (1) Ana Paula Graim Mendonça de Araújo ${ }^{1}$ (1)

1. Universidade do Estado do Pará, Escola de Enfermagem Magalhães Barata, Programa de Pós-Graduação em Enfermagem. Belém, PA, Brasil.

2. Universidade do Estado do Pará, Escola de Enfermagem Magalhães Barata, Departamento de Enfermagem Comunitária. Belém, PA, Brasil.
Autor correspondente:

Fabiane Oliveira da Silva.

E-mail: oliveirafabiane315@gmail.com

Recebido em 17/04/2021.

Aprovado em 06/09/2021.

DOI:https://doi.org/10.1590/2177-9465-EAN-2021-0109

\section{Resumo}

Objetivo: Analisar as percepções de enfermeiros sobre gestão do cuidado e seus fatores intervenientes para o controle da tuberculose na Atenção Primária em Saúde. Método: Estudo descritivo, qualitativo, realizado com 29 enfermeiros que atuavam no controle da tuberculose em 23 Unidades Básicas de Saúde de Belém, Pará. Os dados foram produzidos por entrevistas individuais, utilizando roteiro semiestruturado, e submetidos à análise de conteúdo temática, proposta por Bardin. Resultados: Originaram-se duas categorias temáticas: "A gestão do cuidado de enfermeiros para o controle da tuberculose nas Unidades Básicas de Saúde" e "Fatores intervenientes na efetivação da gestão do cuidado no controle da tuberculose nas Unidades Básicas de Saúde". Conclusão e implicações para a prática: O conhecimento dos enfermeiros sobre a política e a gestão do cuidado no controle da tuberculose precisa ser fortalecido, e embora eles tenham clareza sobre suas competências, não conseguem realizá-las em sua plenitude por questões referentes à pouca organização dos serviços, centralização das atividades nos enfermeiros, baixa cooperação multiprofissional, falta de insumos e de pessoal e questões socioeconômicas ligadas ao usuário com tuberculose.

Palavras-chave: Atenção Primária à Saúde; Enfermagem em Saúde Pública; Gestão em Saúde; Percepção; Tuberculose.

\section{Abstract}

Objective: To analyze nurses' perceptions on care management and its intervening factors for tuberculosis control in Primary Health Care. Method: A descriptive and qualitative study conducted with 29 nurses who worked on tuberculosis control in 23 Basic Health Units in Belém, Pará. The data were produced by individual interviews, using a semi-structured script, and submitted to thematic content analysis, as proposed by Bardin. Results: Two thematic categories emerged, namely: "Care management by nurses for tuberculosis control in Basic Health Units" and "Intervening factors in the application of care management for tuberculosis control in Basic Health Units". Conclusion and implications for the practice: The nurses' knowledge about the care policy and management for tuberculosis control needs to be strengthened and, although nurses are aware of their competences, they are unable to fully implement them due to issues related to poor organization services, centralization of activities on them, low multiprofessional cooperation, scarcity of supplies and personnel, and socioeconomic issues related to tuberculosis patients.

Keywords: Primary Health Care; Public Health Nursing; Health Management; Perception; Tuberculosis.

\section{Resumen}

Objetivo: Analizar la percepción de enfermeros sobre la gestión de la atención y los factores intervinientes para el control de la tuberculosis en la Atención Primaria de la Salud. Método: Estudio descriptivo cualitativo realizado con 29 enfermeros que pre en el control de la tuberculosis en 23 Unidades Básicas de Salud de Belém, Pará. Los datos fueron producidos por entrevistas individuales, utilizando un guion semiestructurado, y sometido al análisis de contenido temático propuesto por Bardin. Resultados: Se originaron dos categorías temáticas: "La gestión de la atención de enfermeros para el control de la tuberculosis en las Unidades Básicas de Salud" y "Factores intervinientes en la efectividad de la gestión de la atención en el control de la tuberculosis en las Unidades Básicas de Salud". Conclusión e implicaciones para la práctica: Es necesario fortalecer el conocimiento de las enfermeras sobre la política y la gestión de la atención en el control de la tuberculosis, y aunque tienen claras sus competencias, no pueden implementarlas en su totalidad debido a problemas relacionados con la mala organización de los servicios, la centralización de actividades en los enfermeros, baja cooperación multiprofesional, falta de insumos y personal y aspectos socioeconómicos relacionados con el usuario con tuberculosis.

Palabras-clave: Atención Primaria a la Salud; Enfermería en Salud Pública; Gestión en Salud; Percepción; Tuberculosis. 


\section{INTRODUÇÃO}

A tuberculose (TB) é uma doença infectocontagiosa, geralmente relacionada à pobreza e aos aglomerados urbanos. Diagnosticar, tratar corretamente e imediatamente os casos de TB pulmonar, forma transmissível, são as principais medidas para seu controle efetivo. No entanto, mesmo com diagnóstico e tratamento disponíveis de forma universal, comumente existem barreiras para acesso aos serviços ${ }^{1}$. Apesar de ser prevenível e curável, a TB ainda é considerada problema de saúde global².

Estima-se que cerca de 10 milhões de pessoas adoeceram por TB no mundo em 2018 e, dessas, 1,5 milhão morreram. No Brasil, foram 4.490 óbitos com coeficiente de mortalidade de 2,2 óbitos/100 mil habitantes. Quanto à incidência, em 2019, foram 73.864 casos novos no Brasil, com coeficiente de incidência de 35,0/100mil habitantes, e em Belém 1.354, com coeficiente de incidência de 90,7/100 mil habitantes ${ }^{3}$.

O Programa Nacional de Controle da Tuberculose (PNCT) é desenvolvido de forma unificada, executado pelas esferas Federal, Estadual e Municipal, com padrões técnicos e assistenciais definidos ${ }^{4}$. Propõe ações de prevenção, controle, tratamento e vigilância dos casos, de forma horizontalizada e descentralizada. Sua implementação é realizada, principalmente, na Atenção Primária à Saúde (APS) ${ }^{5}$.

Nesse cenário, a enfermagem destaca-se no controle da TB, posto que o enfermeiro atua desde a busca de sintomáticos respiratórios até a concretização do diagnóstico. Realiza o Tratamento Diretamente Observado (TDO), monitora os casos confirmados com baciloscopias de controle até o final do tratamento e efetua o controle diário dos faltosos, utilizando estratégias para evitar o abandono. Atua, também, no controle de contatos, tratando, quando indicado, a infecção latente, preenche os instrumentos de vigilância preconizados pelo PNCT (ficha de notificação de caso, livros de registro de sintomáticos respiratórios e de tratamento e acompanhamento dos casos) e realiza atividades de educação, enfatizando a promoção à saúde e prevenção da doença'.

É nesse contexto que se destaca a gestão do cuidado de enfermagem, que contempla seis dimensões: individual, familiar, profissional, organizacional, sistêmica e societária. A dimensão individual é o cuidar de si, no sentido de que cada um tem o poder de produzir um modo singular de fazer suas escolhas, é a mais nuclear de todas as dimensões. A dimensão familiar trata da gestão do cuidado que envolve a relação com pessoas da família, amigos e vizinhos ${ }^{6}$.

A dimensão profissional é regida por três elementos que conferem maior ou menor capacidade de produzir o bom cuidado, como a competência técnica, postura ética e a capacidade de construção de vínculo com quem precisa de seus cuidados ${ }^{6}$.

A dimensão organizacional do cuidado evidencia novos elementos, como o trabalho em equipe, as atividades de coordenação e comunicação, além da função gerencial propriamente dita. A dimensão sistêmica trata da formação de redes ou linhas de cuidado, na perspectiva da construção da integralidade da assistência, e a dimensão societária trata de como, em cada sociedade, se produzem as políticas públicas em geral e de saúde ${ }^{6}$.

Embora existam muitos estudos sobre TB, poucos versam sobre a gestão do cuidado por enfermeiros, mostrando a necessidade de investir nesse tema para a assistência de enfermagem no controle da doença, posto que, geralmente, o enfermeiro está na linha de frente dessas ações nas Unidades Básicas de Saúde (UBS). Nessa perspectiva, a gestão do cuidado se revela como um sistema de cuidados considerando-se diversos aspectos, como: autonomia, individualidade, relações e atitudes profissionais. Compreender um sistema de cuidados remete a várias dimensões com práticas e atitudes que em sua totalidade dão sustentação à dinâmica do cuidado7.

Para o desenvolvimento e aprimoramento da atuação de enfermagem em Saúde Pública, é necessário que os enfermeiros compreendam que o cuidar e o gerenciar constituem a gestão do cuidado integral da enfermagem e devem ser pensados e desenvolvidos em todas as dimensões do cuidado ${ }^{7}$, principalmente no contexto da APS, entendida como eixo estruturante e ordenador da rede de serviços de saúde, com as funções de resolubilidade, coordenação e responsabilização ${ }^{8}$.

Buscando transpor barreiras de acesso e horizontalizar o atendimento aos usuários com TB no Brasil, a descentralização das ações do PNCT para a APS tem sido considerada como arranjo organizacional imprescindível para efetivar o controle da doença, constituindo-se um elemento de destaque nas recomendações adotadas pelo Ministério da Saúde $(\mathrm{MS})^{8}$. Nesse sentido, entende-se que é necessário compreender como se dá a gestão do cuidado de enfermagem no controle da TB e que fatores podem interferir em sua efetivação. Assim, definiu-se como objetivo desta pesquisa: Analisar as percepções de enfermeiros sobre gestão do cuidado e seus fatores intervenientes para o controle da tuberculose na Atenção Primária em Saúde.

\section{MÉTODO}

Pesquisa qualitativa, descritiva, baseada nos critérios consolidados para relato de estudos qualitativos (COREQ) ${ }^{9}$. Foi desenvolvida em 23 das 24 UBS localizadas em Belém, estado do Pará, nas quais o Programa de Controle da Tuberculose (PCT) estava em funcionamento no momento da coleta de dados. Participaram do estudo 29 (93,5\%) enfermeiros, do total de 31, que atuavam no controle da TB nessas Unidades. Incluíramse enfermeiros, com experiência de pelos menos um ano nas ações do PCT nas UBS, sendo que dois enfermeiros optaram em não participar.

A coleta de dados ocorreu no período de novembro de 2018 a março de 2019 por meio de entrevistas individuais, com duração média de 50 minutos, realizadas pela autora principal com recurso de gravação em mídia digital, mediante aceite. Utilizou-se roteiro com questões semiestruturadas, dividido em duas partes: a primeira referente ao perfil dos participantes e a segunda com questões abertas, que exploraram o objeto de estudo.

Inicialmente, apresentou-se o projeto aos enfermeiros das Unidades e realizou-se convite de participação. Isso se deu antes, 
durante ou após o horário de trabalho, de forma a não interferir no pleno exercício de suas atividades. Com os que aceitaram, as entrevistas, foram realizadas no momento da abordagem ou agendadas em dias diferentes, sendo que o local de escolha para todos foi a própria Unidade, em sala reservada, assegurando conforto e privacidade aos entrevistados.

Os depoimentos gravados foram transcritos na íntegra para composição do corpus, posteriormente submetido à análise de conteúdo temática ${ }^{10}$. Foram identificados, predominantemente, nove temas a partir da regularidade com que apareceram nos depoimentos, em ocorrência e co-ocorrência, e mediante suas similaridades foram organizados em duas categorias temáticas.

Os temas referentes aos conhecimentos, às práticas e ações dos enfermeiros compuseram a primeira categoria, intitulada "A gestão do cuidado de enfermeiros para o controle da tuberculose nas Unidades Básicas de Saúde". Por sua vez, os temas que apontaram os fatores intervenientes no contexto do PCT formaram a segunda, com o título "Fatores intervenientes na efetivação da gestão do cuidado no controle da tuberculose nas Unidades Básicas de Saúde". As frequências absolutas e relativas, que indicam a representatividade dos temas, encontramse destacadas ao longo dos resultados da pesquisa.

Atendeu-se à Resolução no 466/12, sendo o projeto aprovado pelo Comitê de Ética em Pesquisa de uma Universidade Pública da cidade de Belém/PA, sob parecer no 2.963.880/CAAE: 98289018.3.0000.5170, em outubro de 2018. Os participantes assinaram o Termo de Consentimento Livre e Esclarecido e o sigilo de suas identidades foi assegurado com o uso de códigos alfanuméricos.

\section{RESULTADOS}

A idade dos participantes variou entre 24 e 61 anos, predominando a faixa etária de 46 a $56(12 / 41,4 \%)$ e o sexo feminino (25/86,2\%). Em relação ao tempo de graduação, $12(41,4 \%)$ declararam de um a dez anos, sete $(24,1 \%)$ de 11 a 20 anos, sete $(24,1 \%)$ de 21 a 30 anos e três $(10,4 \%)$ mais de 30 anos. Entre as especializações, a Saúde Coletiva/Saúde da Família predominou para $11(37,9 \%)$ participantes.

O tempo de atuação nas Unidades variou de um a 21 anos, sendo de um a cinco anos o mais frequente (14/48,3\%). O tempo de atuação no controle da TB variou de um a 30 anos, predominando de um a cinco anos (13/44,8\%). Em relação ao tipo de vínculo empregatício, 24 (82,8\%) eram concursados. Apresentam-se, a seguir, as categorias temáticas originadas a partir da análise.

\section{Categoria 1: A gestão do cuidado de enfermeiros para o controle da tuberculose nas Unidades Básicas de Saúde}

Nesta categoria, discute-se a gestão do cuidado dos enfermeiros para controlar os casos de TB em sua área de atuação, com base em seus conhecimentos sobre a política, práticas e ações programáticas, planejamento em equipe, atividades de educação em saúde na comunidade, acompanhamento dos casos e controle de contatos.
Relativo ao conhecimento a respeito da política e gestão do cuidado, 16 (55,2\%) profissionais manifestaram compreensão sobre aquela, porém muitas respostas trouxeram explicações superficiais ou voltadas somente para a rotina do atendimento nas Unidades, já em relação aos seus conhecimentos sobre esta, 15 (51,7\%) não conseguiram conceituar de forma clara:

Sobre a política em si eu não conheço, só a respeito do programa de TB, do manual, os protocolos a serem seguidos, política mesmo da TB não sei expressar (E1).

Interessante, a gente acaba não observando a gestão do cuidado. O grande problema aqui na Unidade e acredito que em outras Unidades também é a falta de tempo (E26).

Sobre as práticas de cuidados de enfermagem desenvolvidas na UBS, $100 \%$ deles mencionaram as atividades realizadas para acompanhamento dos casos e seus contatos, a maioria embasada nas recomendações dos manuais do MS e protocolos assistenciais:

Aqui a gente faz a consulta de enfermagem, vê o que esse paciente está precisando durante o tratamento, orientações, os exames que ele vai fazer (E2).

Fazemos a solicitação de exames, baciloscopia de escarro, cultura, TRM [Teste Rápido Molecular] e outros exames, detecção de casos novos, dos sintomáticos respiratórios, a convocação de contatos, a prescrição das medicações, conforme o protocolo (E22).

Tratando-se das ferramentas que os profissionais de enfermagem utilizavam nas Unidades para manter o monitoramento dos casos, $100 \%$ deles mencionaram os livros de registro de casos, os boletins e as fichas de aprazamento como os principais instrumentos para esse acompanhamento e avaliação do programa na Unidade:

A gente tem um livro de controle que fica todos os pacientes, aí dá para saber quantos pacientes têm em controle da doença, tem a ficha de aprazamento também que a gente usa para poder saber o dia que o paciente vem consultar, se ele faltou ou não para fazer a busca ativa (E2).

A gente acaba avaliando os nossos livros, boletins mensais, porque o boletim é um documento que dá uma visão geral onde estamos falhando ou onde podemos melhorar (E6).

Em relação às atividades de planejamento, ao aprimoramento do monitoramento dos casos, à avaliação e ao trabalho em equipe, 16 (55,2\%) afirmaram que buscavam reunir e pactuar conjuntamente com a equipe multiprofissional, no entanto, havia o reconhecimento que esse esforço era, na maioria das vezes, infrutífero, pois não existia periodicidade dessas reuniões, além da falta de envolvimento de algumas categorias profissionais nesses planejamentos: 
Nós temos meses específicos que reunimos sobre determinada doença. A gestão chama e nós programamos, sempre um cobrando o outro a respeito das orientações aos usuários, mas nem toda a equipe se envolve $e$ participa (E15).

As reuniões são realizadas somente quando necessário, não tem uma periodicidade creio que mais por falta de tempo mesmo, porque a Unidade é muito procurada e os profissionais acabam ficando sem tempo para realizar as reuniões de forma periódica (E24).

Quanto às atividades de educação em saúde, com intuito de informar e orientar usuários com TB e familiares para melhoria da adesão e acompanhamento ao tratamento, 15 (51,7\%) afirmaram que realizavam atividades educativas com a comunidade por meio de palestras, dramatizações, rodas de conversa, entre outras, vistas como fundamentais para a sensibilização da clientela porém não havia periodicidade e eram realizadas em épocas específicas do ano, a exemplo de campanhas:

Em época de campanha nós realizamos palestras educativas para os pacientes (E23).

Realizo palestra e peça [teatral] também, sempre a gente está fazendo! A gente faz onde "o povão" está, dia de terça-feira geralmente, que é o dia que o médico vem, como ele atende muita gente é mais cheio, e eles gostam muito de peça, porque prende, chama mais atenção (E28).

A participação da família para adesão do usuário com TB ao tratamento e controle da doença foi considerada importante, uma vez que 10 (34,5\%) mencionaram que buscavam orientar e atrair tanto a eles quanto seus familiares, não apenas objetivando avaliar os contatos domiciliares, mas também melhorar a aderência às ações de controle, pois a família configurava-se como um apoio importante ao PCT:

O paciente costuma aderir muito bem ao tratamento, vem nas consultas e toma a medicação quando a família está perto para ajudar, claro que tem casos muito complicados, mas, no geral, a gente tem esse apoio (E22).

Não temos Estratégia Saúde da Família na nossa área e aí fica difícil, pois nós não temos essa busca ativa, mas sempre pegamos o contato de algum parente da família e ligamos atrás deles quando faltam muito, é o que podemos fazer, contar com a ajuda da família mesmo (E17).

\section{Categoria 2: Fatores intervenientes na efetivação da gestão do cuidado no controle da tuberculose nas Unidades Básicas de Saúde}

Nesta categoria, apresentam-se os fatores que interferiam na efetivação do programa de controle da TB nas UBS. Conforme os depoimentos, estavam relacionados principalmente aos serviços para 27 (93,1\%), e aos usuários com TB para $25(86,2 \%)$ dos participantes.

No contexto dos serviços, em relação às facilidades e dificuldades, destacou-se a relação com a gerência local (direção da UBS), responsável pelo serviço da Unidade e sua articulação com o município, e gerência central (Coordenação Municipal de controle da TB).

A maioria, 27 (93,2\%) (93,1\%), afirmou que tinha boa relação e discutia sobre as práticas relacionadas ao controle da TB com a gerência local, facilitando o bom andamento das ações nas Unidades. Já com a gerência central, 24 (82,8\%) afirmaram ter dificuldades, pois não havia, até o momento, um coordenador municipal no programa de TB, contando nessa circunstância apenas com os técnicos que, embora mostrassem boa vontade, muitas vezes, não conseguiam atender às demandas:

A gerente daqui é muito atenciosa e participativa, se preocupa com a falta de materiais e está sempre querendo o retorno da equipe (E28).

Dificuldade eu acho que tem mais na questão central, por exemplo, agora a referência de tuberculose e hanseníase no município está sem coordenador, acho que já tem mais de 1 ou 2 meses que está sem coordenação (E19).

Também foi mencionado como problema a centralização em algumas Unidades dos exames para diagnóstico e controle, que implicava em encaminhar as amostras de escarro e receber os resultados por meio de um serviço específico chamado "rota", que algumas vezes atrasava, retardando o início do tratamento e acarretando prejuízo para usuário com TB e comunidade, principalmente nos casos positivos da doença:

Hoje em dia, "a rota" vem buscar uma vez na semana $e$ às vezes nem vem, isso atrasa muito o diagnóstico do paciente e o início do tratamento, principalmente os positivos para a doença (E14).

Agora nós estamos tendo problema com a demora do diagnóstico, a gente faz o pedido, alguém sempre vem buscar, mas o retorno é muito demorado e acaba que as coisas tendem a não funcionar de forma mais eficiente (E19).

Ao serem perguntados sobre as reuniões com gestores e equipe para aprimoramento das ações em prol da melhoria do serviço e do atendimento prestado, 13 (44,8\%) afirmaram que não eram realizadas e, embora $12(41,4 \%)$ tenham mencionado que as reuniões aconteciam, todos reconheciam que estas não tinham periodicidade, em geral, pela falta de tempo devido à grande demanda de atendimento, pela falta de espaço físico para tal, falta de planejamento e organização, bem como pelo pouco interesse da maioria:

Reunião não! A gente acaba conversando individualmente (E6). 
Não são realizadas com frequência e o motivo acredito que seja a falta de tempo e falta também um planejamento a respeito disso, pois se houvesse planejamento, haveria tempo para reunir (E11).

Sobre as atualizações e capacitações ofertadas relativas à política, para seu aprimoramento teórico e profissional, 19 (65,5\%) participantes afirmaram que não havia periodicidade nas capacitações e atualizações sobre TB desde que ingressaram no serviço, assim como havia dificuldade de se afastar das atividades na Unidade quando convocados, uma vez que existia uma alta demanda de atendimento:

De vez em quando temos capacitações, mas não tem uma frequência regular (E6).

Nunca mais foram realizadas e, quando têm, nós temos dificuldade de sair da Unidade, porque não tem quem substituir e o gerente fica na "corda bamba", já que o serviço para e a demanda de pacientes é grande (E14).

Por meio das falas, percebeu-se a centralização das ações de controle nos enfermeiros, uma vez que $22(75,9 \%)$ afirmaram a pouca adesão dos demais profissionais, em especial do médico, e a recusa de alguns em atender aos usuários com TB devido à falta de conhecimento, ao estigma e medo em relação à doença, problemas que, muitas vezes, são agravados pela baixa frequência de capacitações sobre TB e, em geral, quando estas aconteciam, apenas a equipe de enfermagem comparecia:

A dificuldade é que ainda existe na mente do profissional muito medo em relação à TB. Temos médicos que ficam desesperados quando vão atender um paciente com $T B(E 4)$.

A equipe de enfermagem tem dificuldade para colocar em prática as ações em decorrência do excesso de trabalho e atribuições, os profissionais das demais áreas não são bem preparados para atender aos pacientes com $T B$, assim como muitos têm preconceito e medo (E7).

Ficaram evidentes as dificuldades que eles enfrentavam nas Unidades para manterem as ações de controle da TB. Nesse sentido, 15 (51,7\%) mencionaram a falta de insumos e de pessoal como principais entraves:

Pelo excesso de trabalho, poucos profissionais estão atendendo. Se tivesse mais enfermeiros para assumir o programa, iria andar com mais eficiência (E7).

Teve um determinado período que faltou medicamento, mas a gente soube ter jogo de cintura, dá um pouquinho para um, um pouquinho para outro, até chegar o restante da medicação (E28).

Em relação aos usuários com TB, as dificuldades mais relatadas referem-se à questão socioeconômica e suas consequências, caracterizadas, principalmente, pelos dependentes químicos (usuários de drogas lícitas e ilícitas) e moradores de rua, assim como dificuldades para deslocamento até à Unidade, ocasionando a falta e tornando-os mais suscetíveis ao abandono do tratamento, dificultando o controle da doença, como mencionado por $20(69 \%)$ participantes:

Há dificuldade na questão social, de abandono de tratamento, usuários de drogas, pacientes que mudam de endereço e perdemos o contato, e aqui nesse bairro essa questão social é muito forte, por isso, infelizmente, há muito abandono (E15).

Em relação ao paciente, o que acaba apresentando um pouco mais de problema é exatamente quando nós temos o TDO e não tem recurso, não tem nada a ver com vínculo, com a Unidade, e sim com a questão social (E19).

\section{DISCUSSÃO}

Para desenvolver a gestão do cuidado integral em enfermagem, o enfermeiro deve utilizar ferramentas de gestão como indicadores de saúde, planejamento de materiais e recursos humanos, normas de segurança para atendimento, processo de tomada de decisão, entre outras. Para isso, o cuidado prestado diretamente ao público deve englobar técnicas, tecnologias, procedimentos e ações de prevenção, promoção e educação em saúde ${ }^{7}$.

Destaca-se que os profissionais de saúde que atuam na gestão do cuidado precisam ser preparados para essa atividade em todas as suas dimensões, de modo que compreendam seu papel e estejam capacitados para acompanhar os usuários com TB, demonstrando a eles que podem e devem atuar em prol da própria saúde, com ações que visam sua promoção emanutenção. ${ }^{7}$

Nesse contexto, quanto aos conhecimentos a respeito da política e da gestão do cuidado, os enfermeiros desse estudo demonstraram compreensão sobre seus conceitos, porém um tanto superficial ou voltada somente para a rotina do atendimento nas Unidades. Essa falta de conhecimento mais aprofundado sobre os temas pode estar intimamente ligada à pouca capacitação dos profissionais.

Nesse sentido, estudo realizado na África do Sul ${ }^{11}$ demonstrou que é de suma importância que as práticas de controle da TB estejam influenciadas por fatores-chave, como conhecimento aprofundado e ações de prevenção, no entanto, na realidade da APS, ainda identificam-se enfermeiros com baixo conhecimento e práticas inadequadas de controle e prevenção da TB, capazes de afetar a assistência prestada.

Outra pesquisa realizada em Divinópolis, MG, evidenciou que o PCT local apresentava como dificuldades a fragilidade do planejamento, inexistência de um modelo de difusão de informações e insuficiência de capacitações e profissionais treinados que, por sua vez, demonstraram falta de conhecimento em áreas como vigilância, diagnóstico e realização do TDO ${ }^{12}$. Assim, entende-se que a educação permanente é estratégia de 
suma importância, pois amplia o conhecimento teórico sobre os elementos que estão aliados diretamente à prática.

Uma vez empoderados dos conhecimentos necessários, os enfermeiros tornam-se adequadamente preparados para o planejamento do controle da TB. No Brasil, o PNCT busca aprimorar a qualificação do planejamento e a descentralização das ações de controle para ampliar o acesso dos doentes aos serviços de saúde ${ }^{13}$. É o que reforça estudo realizado na província de Tak, Tailândia, que apontou o planejamento como um dos elementos principais para o controle da TB, juntamente com o compartilhamento eficaz de informações, a melhoria no diagnóstico, entre outros ${ }^{14}$.

Assim, entende-se a educação em saúde como um dos elementos primordiais para o planejamento de enfermagem, a fim de orientar usuários com TB e familiares, bem como melhorar a adesão ao tratamento. Neste estudo, esta mostrou-se presente na rotina das Unidades, porém com pouca periodicidade ou somente em época de campanhas, o que precisaria ser modificado para alcance de melhores resultados. Uma pesquisa sobre representações sociais de profissionais de saúde e usuários com TB mostrou que a educação em saúde tem reflexo positivo para a adesão ao tratamento, pois é ferramenta para reduzir as chances de abandono, formando consciência crítica e favorecendo o poder de escolhas saudáveis de vida ${ }^{15}$.

A rede de apoio familiar e social torna-se extremamente necessária para esses usuários, estando positivamente associada a comportamentos de busca de saúde, adesão ao tratamento e resultados satisfatórios de saúde, podendo a família atuar como um amortecedor de estresse para o seu bem-estar psicossocial ${ }^{16}$.

Estudo realizado no Paquistão ${ }^{17}$ evidenciou que quando os usuários com TB eram tratados com cuidado e observados pela família e pela comunidade ao redor, maiores taxas de adesão ao tratamento e maior bem-estar psicológico do usuário eram constatados, revelando que essa rede de apoio é benéfica para a saúde, uma vez que esses usuários apresentam necessidades biológicas e não biológicas que devem ser atendidas para a cura da doença e se eles não recebem suporte pela família e comunidade, isso pode levar à não adesão ao tratamento.

A partir desse entendimento, a consulta de enfermagem pode ser utilizada como um instrumento desse processo, pois permite o estreitamento da relação com o usuário com TB e família e o planejamento do seu cuidado. Seu potencial como estratégia para o cuidado efetivo oferece vantagens, auxiliando na identificação de problemas, necessidades, tomada de decisão, planejamento, condutas e o enfrentamento de outras demandas cotidianas desse usuário. Assim, evita-se a fragmentação do cuidado, buscando, na medida do possível, sanar suas necessidades ${ }^{18}$.

Ressalta-se também que, para o cuidado integral do usuário com TB, a participação da equipe interdisciplinar para o desenvolvimento das ações nas Unidades é imprescindível. No entanto, conforme descrito pelos enfermeiros, existia pouca cooperação dos demais membros da equipe multiprofissional, em especial, dos médicos. Nesse contexto, as ações de cuidado à TB requerem abordagem interdisciplinar, de forma coordenada, operacionalização baseada em relações que envolvam pessoas, tecnologias, recursos e responsabilidade compartilhada, considerando que todos os envolvidos no processo têm a mesma importância ${ }^{19}$.

Estudo realizado nas Unidades de Saúde da Família do município do Rio de Janeiro, $\mathrm{RJ}^{20}$, também identificou que a maior parte dos enfermeiros dessas Unidades participou de capacitações sobre TB, o que demonstrou maior interesse na temática ou mais disponibilidade. Já a falta ou a alta rotatividade de médicos nos serviços aumentou as demandas de atendimentos e limitou a participação desses profissionais nas capacitações.

Essa cooperação reduzida da equipe multiprofissional pode explicar porque identificou-se nos depoimentos a centralização das ações de controle da TB nos enfermeiros. Estudo que abordou fragilidades e limites das práticas dos enfermeiros na APS no Brasil também apontou dificuldades relativas à falta de recursos humanos com centralização das atividades no enfermeiro, mostrando a necessidade de articulação de uma gestão de processos compartilhada com a equipe multiprofissional, para que o trabalho dos enfermeiros seja realizado com efetividade nas dimensões do cuidado e da gestão em saúde nas Unidades da APS $^{21}$.

Nessa perspectiva, entendendo que há uma relação simbiótica entre os recursos, as estruturas e o modelo de gestão em saúde, é fundamental que os gestores tenham a capacidade de realizar uma leitura situacional dinâmica e precisa do contexto do sistema de saúde ${ }^{22}$. Daí a importância das gerências local e central estarem frequentemente em contato com os profissionais das Unidades, por meio de reuniões contínuas ou visitas técnicas, dimensionando os problemas existentes e buscando resolução para os fatores intervenientes da gestão do cuidado integral de enfermagem em parceria com as equipes no contexto da TB.

É fato que problemas estruturais e logísticos relacionados aos serviços podem comprometer o envolvimento da equipe de saúde com as ações de controle da TB e, consequentemente, a resolutividade e qualidade dos serviços oferecidos, mostrando que profissionais, gerência local e central, assim como neste estudo, precisam ser qualificados para atuar na gestão do cuidado integral junto aos doentes, devendo ser estabelecida maior integração dos serviços de saúde, fluxograma de atendimento para o acesso às tecnologias necessárias e o monitoramento dos usuários com TB, no sentido de assegurar o cuidado apoiado e integral ${ }^{23}$.

Estudo abrangendo municípios brasileiros mostrou que a adequada provisão de insumos e recursos humanos, bem como a solicitação de baciloscopia de escarro, estão associadas positivamente à detecção de casos em serviços da APS ${ }^{24}$. Isso permite entender por que a falta de insumos e de pessoal foi associada, neste estudo, a problemas para o acompanhamento adequado dos casos de TB.

Além desses fatores intervenientes para o controle da doença, também se apontou para o estigma da doença ainda presente, dificultando encaminhamentos e resolutividade para avaliar e sanar problemas. A qualidade do cuidado e o sucesso do tratamento perpassam inúmeros aspectos da relação entre o doente e as equipes de saúde, ou seja, o cuidado requer que o profissional converta a dimensão econômica e técnica de seu trabalho em dimensão relacional e compreensiva ${ }^{25}$. 
As atitudes tomadas pelos profissionais que resultam em comportamentos estigmatizantes, relacionados aos doentes, se desvelam por ações discriminatórias advindas daqueles que deveriam prestar o cuidado. Essa realidade cria barreiras no acesso aos serviços de saúde e influencia no diagnóstico e tratamento da TB, agravando ainda mais a realidade dessas pessoas ${ }^{26}$. Vale ressaltar a importância de postura ética que perpassa por não demonstrar qualquer rejeição à pessoa doente, por medo de contrair a doença, o que poderia impactar no vínculo e adesão desse usuário com TB ao tratamento ${ }^{19}$.

As falas dos enfermeiros referentes às dificuldades enfrentadas quanto à questão socioeconômica e suas consequências, como a dependência química de drogas lícitas e ilícitas e situação de rua, deixam evidentes as vulnerabilidades sociais que estão intimamente ligadas a TB. Esses achados também foram encontrados em estudo realizado em 645 municípios do estado de São Paulo, demonstrando que usuários de droga e moradores de rua são socialmente excluídos e vulneráveis, apresentam conflitos emocionais e psicológicos agravados pela falta de apoio familiar, situações que implicam diretamente no tratamento, acarretando sucessivos abandonos e o desenvolvimento da TB multirresistente, pois o vínculo entre profissional e usuário com TB é enfraquecido, sendo este primordial para o sucesso do tratamento ${ }^{27}$.

Estudo realizado no Cazaquistão $0^{28}$ mostrou que o encarceramento, a dependência de álcool e outras drogas, o diabetes e o baixo acesso à assistência médica estavam associados à maior índice de casos de TB pulmonar positiva, devendo as políticas de ação, tratamento e prevenção levar em consideração esses fatores para conter a doença. Deve-se reconhecer e valorizar as situações de vulnerabilidades em que se encontra o usuário com TB para melhor assisti-lo e protegê-lo, pois a análise da vulnerabilidade permite conhecer e compreender as diferenças como cada um vivencia e enfrenta o processo saúde-doença ${ }^{29}$.

Portanto, para que se consiga enfrentar os fatores intervenientes na gestão do cuidado, necessita-se da busca por novos modelos bem concebidos e integrados na APS para o acesso ao tratamento da TB, aumento da notificação de casos e melhor adesão ao plano terapêutico estabelecido, construído diante da compreensão do indivíduo como um todo, plural e complexo. Para tanto, a integração bem sucedida do serviço em torno do controle da TB deve incluir disponibilidade material e treinamento especializado de recursos humanos, aliada a competência gerencial e supervisão do serviço de $\mathrm{TB}^{30}$.

\section{CONCLUSÃO E IMPLICAÇÕES PARA A PRÁTICA}

A descrição da gestão do cuidado e a identificação dos fatores que interferem para o controle da TB na Atenção Primária permitiu identificar que o conhecimento dos enfermeiros sobre a política e a gestão do cuidado no controle da TB precisa ser fortalecido, pois essa fragilidade dificulta o controle da doença na APS, sendo a falta de capacitação um fator potencializador desse cenário. Embora os enfermeiros tivessem clareza sobre suas competências, não conseguiam realizá-las em sua plenitude por questões referentes à pouca organização dos serviços, centralização das atividades nos enfermeiros, baixa cooperação multiprofissional, falta de insumos e de pessoal e questões socioeconômicas ligadas ao usuário com TB.

As dimensões da gestão do cuidado (individual, familiar, profissional, organizacional, sistêmica e societária) estavam fragilizadas, pois as estratégias eram insuficientes para o controle da doença e impactavam diretamente na qualidade do cuidado prestado aos usuários com TB. São aspectos que precisam ser resolutivos, merecem olhar mais crítico dos profissionais envolvidos e dos gestores, em prol de seu efetivo controle no município.

Dadas as lacunas nas publicações acerca da gestão do cuidado na política de controle da TB, a discussão sobre o tema pode ter trazido limitações ao estudo. Outra limitação é o fato do estudo ter contado somente com participação dos profissionais ligados à assistência, sendo necessária também a participação de gestores para ampliação da discussão sobre o tema. Ainda assim, entende-se que este estudo pode contribuir para estimular a implementação de estratégias para o controle da TB, que considerem o contexto da gestão do cuidado de enfermagem, evidenciada como pouco valorizada, além de subsidiar a realização de novas pesquisas.

Espera-se que os achados desta pesquisa suscitem reflexões acerca da gestão do cuidado para o controle da TB na APS, partindo do princípio de que ela é uma ferramenta necessária para a atenção integral ao usuário com TB e à comunidade. Destacase, portanto, o papel gerencial e educador da enfermagem em Saúde Pública, considerada, apesar das dificuldades, como referência e indispensável para o acompanhamento dos casos.

\section{CONTRIBUIÇÕES DOS AUTORES}

Desenho do estudo: Fabiane Oliveira da Silva. Ivaneide Leal Ataíde Rodrigues.

Coleta ou produção dos dados: Fabiane Oliveira da Silva.

Análise de dados: Fabiane Oliveira da Silva. Ivaneide Leal

Ataíde Rodrigues. Alexandre Aguiar Pereira.

Interpretação dos resultados: Fabiane Oliveira da Silva. Ivaneide Leal Ataíde Rodrigues. Alexandre Aguiar Pereira. Laura Maria Vidal Nogueira. Erlon Gabriel Rego de Andrade.

Redação e revisão crítica do manuscrito: Fabiane Oliveira da Silva. Ivaneide Leal Ataíde Rodrigues. Alexandre Aguiar Pereira. Laura Maria Vidal Nogueira. Erlon Gabriel Rego de Andrade. Ana Paula Graim Mendonça de Araújo.

Aprovação da versão final do artigo: Fabiane Oliveira da Silva. Ivaneide Leal Ataíde Rodrigues. Alexandre Aguiar Pereira. Laura Maria Vidal Nogueira. Erlon Gabriel Rego de Andrade. Ana Paula Graim Mendonça de Araújo.

Responsabilidade por todos os aspectos do conteúdo e a integridade do artigo publicado: Fabiane Oliveira da Silva Ivaneide Leal Ataíde Rodrigues. Alexandre Aguiar Pereira. Laura Maria Vidal Nogueira. Erlon Gabriel Rego de Andrade. Ana Paula Graim Mendonça de Araújo. 


\section{EDITOR ASSOCIADO}

\section{Maria Catarina Salvador da Motta (1)}

\section{EDITOR CIENTÍFICO}

\author{
Ivone Evangelista Cabral (B)
}

\section{REFERÊNCIAS}

1. Ministério da Saúde (BR), Secretaria de Vigilância em Saúde, Departamento de Vigilância Epidemiológica. Manual de Recomendações para o Controle da Tuberculose no Brasil [Internet]. Brasília (DF): Ministério da Saúde; 2018 [citado 2021 fev 21]. Disponível em: http://bvsms. saude.gov.br/bvs/publicacoes/manual_recomendacoes_controle_ tuberculose_brasil_2_ed.pdf

2. Andrade KVF, Nery JS, Souza RA, Pereira SM. Effects of social protection on tuberculosis treatment outcomes in low or middle-income and in high-burden countries: systematic review and meta-analysis. Cad Saude Publica. 2018;34(1):e00153116. http://dx.doi.org/10.1590/0102311x00153116. PMid:29412320.

3. Ministério da Saúde (BR), Secretaria de Vigilância em Saúde, Departamento de Vigilância Epidemiológica. Boletim epidemiológico de tuberculose 2020 [Internet]. Brasília (DF): Ministério da Saúde; 2020 [citado 2021 fev 12]. Disponível em: http://www.aids.gov.br/pt-br/ pub/2020/boletim-epidemiologico-de-turbeculose-2020

4. Shuhama BV, Silva LMC, Andrade RLP, Palha PF, Hino P, Souza KMJ. Avaliação do tratamento diretamente observado da tuberculose segundo dimensões da transferência de políticas. Rev Esc Enferm USP. 2017;51:e03275. http://dx.doi.org/10.1590/s1980-220x2016050703275. PMid:29267738.

5. Medeiros ER, Silva SYB, Ataide CAV, Pinto ESG, Silva MLC, Villa TCS. Clinical information systems for the management of tuberculosis in primary health care. Rev Lat Am Enfermagem. 2017;25(0):e2964. http://dx.doi.org/10.1590/1518-8345.2238.2964. PMid:29236840.

6. Cecilio LCO. Apontamentos teórico-conceituais sobre processos avaliativos considerando as múltiplas dimensões da gestão do cuidado em saúde. Interface (Botucatu). 2011 jun;15(37):589-99. http://dx.doi. org/10.1590/S1414-32832011000200021.

7. Siewert JS, Rodrigues DB, Malfussi LBH, Andrade SR, Erdmann AL. Management of integral care in nursing: reflections under the perspective of complex thinking. REME - Rev Min Enferm. 2017;21:e-1047. http:// dx.doi.org/10.5935/1415-2762.20170057.

8. Wysocki AD, Ponce MAZ, Brunello MEF, Beraldo AA, Vendramini SHF, Scatena LM et al. Atenção Primária à Saúde e tuberculose: avaliação dos serviços. Rev Bras Epidemiol. 2017 mar;20(1):161-75. http://dx.doi. org/10.1590/1980-5497201700010014. PMid:28513803.

9. Souza VR, Marziale MH, Silva GT, Nascimento PL. Tradução e validação para a língua portuguesa e avaliação do guia COREQ. Acta Paul Enferm. 2021;34:eAPE02631. http://dx.doi.org/10.37689/acta-ape/2021AO02631.

10. Bardin L. Análise de conteúdo. São Paulo: Edições 70; 2016.

11. van Rensburg AJ, Engelbrecht M, Kigozi G, van Rensburg D. Tuberculosis prevention knowledge, attitudes, and practices of primary health care nurses. Int J Nurs Pract. 2018 dez;24(6):e12681. http://dx.doi. org/10.1111/ijn.12681. PMid:30066350.

12. Andrade HS, Oliveira VC, Gontijo TL, Pessôa MTC, Guimarães EAA. Avaliação do Programa de Controle da Tuberculose: um estudo de caso. Saúde Debate. 2017 mar;41(spe):242-58. http://dx.doi.org/10.1590/0103$11042017 s 18$.

13. Pinheiro PGOD, Sá LD, Palha PF, Oliveira RCC, Nogueira JA, Villa TCS. Critical points for the control of tuberculosis on Primary Health Care. Rev Bras Enferm. 2017 dez;70(6):1227-34. http://dx.doi.org/10.1590/00347167-2016-0467. PMid:29160484.

14. Tschirhart N, Thi SS, Swe LL, Nosten F, Foster AM. Treating the invisible: gaps and opportunities for enhanced TB control along the ThailandMyanmar border. BMC Health Serv Res. 2017 jan 13;17(1):29. http:// dx.doi.org/10.1186/s12913-016-1954-9. PMid:28086877.
15. Oliveira RA, Lefèvre F. Communication on disclosure of tuberculosis diagnosis and adherence to treatment: social representations of professionals and patients. Texto Contexto Enferm. 2017;26(2):e06790015. http://dx.doi.org/10.1590/0104-07072017006790015.

16. van Hoorn R, Jaramillo E, Collins D, Gebhard A, van den Hof S. The effects of psycho-emotional and socio-economic support for tuberculosis patients on treatment adherence and treatment outcomes - A Systematic Review and Meta-Analysis. PLoS One. 2016 abr 28;11(4):e0154095. http://dx.doi.org/10.1371/journal.pone.0154095. PMid:27123848.

17. Saqib SE, Ahmad MM, Panezai S. Care and social support from family and community in patients with pulmonary tuberculosis in Pakistan. Fam Med Community Health. 2019 out 30;7(4):e000121. http://dx.doi. org/10.1136/fmch-2019-000121. PMid:32148725.

18. Kahl C, Meirelles BHS, Lanzoni GMM, Koerich C, Cunha KS. Ações e interações na prática clínica do enfermeiro na Atenção Primária à Saúde. Rev Esc Enferm USP. 2018;52:e03327. http://dx.doi.org/10.1590/ s1980-220x2017025503327. PMid:29846488.

19. Melo LSO, Oliveira EN, Neto FRGX, Viana LS, Prado FA, Costa JBC Passos e descompassos no processo de cuidado aos portadores de tuberculose na Atenção Primária. Enferm Foco [Internet]. 2020 [citado 2021 fev 4];11(1):136-41. Disponível em: http://revista.cofen.gov.br/ index.php/enfermagem/article/view/2917/718

20. Barros RSL, Mota MCS, Abreu AMM, Villa TCS. Desempenho do programa de controle da tuberculose na estratégia saúde da família. Esc Anna Nery. 2020;24(4):e02020002. http://dx.doi.org/10.1590/21779465-ean-2020-0002.

21. Ferreira SRS, Périco LAD, Dias VRGF. The complexity of the work of nurses in Primary Health Care. Rev Bras Enferm. 2018;71(Suppl 1):7049. http://dx.doi.org/10.1590/0034-7167-2017-0471. PMid:29562031.

22. Soder R, Oliveira IC, Da Silva LAA, Santos JLG, Peiter CC, Erdmann AL. Desafios da gestão do cuidado na Atenção Básica: perspectiva da equipe de enfermagem. Enferm Foco. 2018;9(3):76-80. http://dx.doi. org/10.21675/2357-707X.2018.v9.n3.1496.

23. Oliveira AH, Pinto AGA, Lopes MSV, Figueiredo TMRM, Cavalcante EGR. Therapeutic itinerary of people with tuberculosis in face with their health needs. Esc Anna Nery. 2019;23(3):e20190034. http://dx.doi. org/10.1590/2177-9465-ean-2019-0034.

24. Pelissari DM, Bartholomay P, Jacobs MG, Arakaki-Sanchez D, Anjos DSO, Costa MLS et al. Oferta de serviços pela atenção básica e detecção da incidência de tuberculose no Brasil. Rev Saude Publica. 2018 maio 3;52:53. http://dx.doi.org/10.11606/S1518-8787.2018052000131. PMid:29791528.

25. Ferreira JT, Engstrom EM. Estigma, medo e perigo: representações sociais de usuários e/ou traficantes de drogas acometidos por tuberculose e profissionais de saúde na Atenção Básica. Saude Soc. 2017 dez;26(4):1015-25. http://dx.doi.org/10.1590/s0104-12902017155759.

26. Hino P, Monroe AA, Takahashi RF, Souza KMJ, Figueiredo TMRM, Bertolozzi MR. Tuberculosis control from the perspective of health professionals working in street clinics. Rev Lat Am Enfermagem. 2018;26(0):e3095. http://dx.doi.org/10.1590/1518-8345.2691.3095. PMid:30517582.

27. Arroyo LH, Ramos ACV, Yamamura M, Berra TZ, Alves LS, Belchior AS et al. Predictive model of unfavorable outcomes for multidrug resistant tuberculosis. Rev Saude Publica. 2019;53:77. http://dx.doi. org/10.11606/s1518-8787.2019053001151. PMid:31553380.

28. Hermosilla S, You P, Aifah A, Abildayev T, Akilzhanova A, Kozhamkulov $U$ et al. Identifying risk factors associated with smear positivity of pulmonary tuberculosis in Kazakhstan. PLoS One. 2017 mar 1;12(3):e0172942. http://dx.doi.org/10.1371/journal.pone.0172942. PMid:28249005.

29. Janssens JP, Wuillemin T, Adler D, Jackson Y. Screening for tuberculosis in an urban shelter for homeless in Switzerland: a prospective study. BMC Infect Dis. 2017 maio 16;17(1):347. http://dx.doi.org/10.1186/ s12879-017-2449-y. PMid:28511638.

30. Chikovani I, Diaconu K, Duric P, Sulaberidze L, Uchaneishvili M, Mohammed NI et al. Addressing challenges in tuberculosis adherence via performance-based payments for integrated case management: protocol for a cluster randomized controlled trial in Georgia. Trials. 2019 ago 28;20(1):536. http://dx.doi.org/10.1186/s13063-019-3621-z. PMid:31462284. 